\title{
Características histológicas de la tumefacción frontal de Melanophryniscus (Amphibia: Anura: Bufonidae)
}

\author{
Histological features of the frontal tumefaction in Melanophryniscus \\ (Amphibia: Anura: Bufonidae)
}

\author{
DANIEL E. NAYA ${ }^{1,2 *}$, JOSÉ A. LANGONE ${ }^{3} \&$ RAFAEL O. DE SÁ ${ }^{4}$ \\ ${ }^{1}$ Sección Zoología Vertebrados. Facultad de Ciencias. Universidad de la República, Iguá 4225, \\ Montevideo 11400, Uruguay \\ ${ }^{2}$ Dirección actual: Center for Advanced Studies in Ecology and Biodiversity \& Departamento de Ecología, Pontificia \\ Universidad Católica de Chile, Santiago 6513677, Chile \\ ${ }^{3}$ Departamento de Herpetología, Museo Nacional de Historia Natural y Antropología, Casilla de Correo 399, \\ Montevideo 11000, Uruguay; e-mail: pplangone@fcien.edu.uy \\ ${ }^{4}$ Department of Biology, University of Richmond, Richmond, Virginia 23173, USA; e-mail: rdesa@richmond.edu. \\ *Autor para correspondencia: e-mail: dnaya@bio.puc.cl
}

\begin{abstract}
RESUMEN
Se analizó la histología tegumentaria de la región cefálica en seis especies del género Melanophryniscus, pertenecientes a los grupos stelzneri (4) y tumifrons (2), con el objetivo de esclarecer la estructura de la tumefacción frontal y su posible función. Las características generales de la histología del grupo stelzneri, coincidieron con las descripciones tegumentarias existentes para el género. Sin embargo, la región cefálica frontal en las dos especies del grupo tumifrons muestran cambios histológicos correlacionados a la tumefacción frontal. Esta estructura presenta una organización similar a otras macroglándulas presentes en la dermis de anuros (e.g., parotoides, paracnémicas), y por tanto se propone el nombre de macroglándula frontal para la misma. Los resultados obtenidos sugieren un rol defensivo para esta macroglándula.
\end{abstract}

Palabras clave: glándulas, histología, Melanophryniscus, piel, tegumento.

\begin{abstract}
We analyzed the histology of skin from the head region of six species of Melanophryniscus, belonging to the stelzneri (4) and tumifrons (2) groups, to understand the frontal tumefaction structure and function. The histological features of the skin in the stelzneri group are in agreement with the current description for the genus Melanophryniscus. However, the features of the skin on the anterior head region of M. tumifrons shows several changes associated with the frontal tumefaction. This structure consists of a macrogland of similar organization to that of other dermic glands (e.g., parotoid, paracnemid) of amphibians. We propose the name frontal macrogland for this structure. Our results suggest a possible defense role for this macrogland.
\end{abstract}

Key words: glands, histology, Melanophryniscus, skin, tegument.

\section{INTRODUCCIÓN}

La colonización de nuevos ambientes suele resultar en presiones selectivas que favorecen una rápida divergencia en relación al linaje ancestral (Price et al. 2003). Para los anfibios la colonización de ambientes terrestres determinó profundas modificaciones organísmicas, tanto a nivel morfológico como fisiológico y comportamental. Algunos de los cambios más notorios se refieren a las características del tegumento, el cual elevó su diversidad morfofuncional pasando a tomar parte en diversos procesos tales como la respiración, reproducción, defensa y protección contra la desecación (Toledo \& Jared 1993). Como consecuencia de esto los anfibios actuales presentan una alta dependencia de su tegumento (Toledo \& Jared 1995), lo que a su vez ha incentivado el estudio de las características tegumentarias en dicho grupo (Heatwole \& Barthalmus 1994). 
Para el caso particular del género Melanophryniscus la histología de su tegumento ha sido analizada por diversos autores. Biraben (1929) refiriéndose a Atelopus stelzneri (probablemente $M$. s. stelzneri) es el primero en afirmar que la piel de esta especie posee dos tipos de glándulas (mucosas y granulares) cuya estructura es similar a la de otras especies de anuros. Santos (1979) realizó una descripción detallada de la piel dorsal de $M$. stelzneri atroluteus (actualmente $M$. atroluteus). Más recientemente, Mangione \& Alcaide (1994) analizaron las diferencias histológicas del parche pélvico en referencia a piel dorsal y ventral en $M$. rubiventris subconcolor, mientras que Delfino et al. (1998) describieron el dimorfismo de las glándulas granulares en ejemplares de $M$. stelzneri (= M. cupreuscapularis). Sin embargo, al presente ningún trabajo ha analizado la histología de la tumefacción frontal, estructura anatómica localizada en el extremo dorsal de la cabeza, que ha sido usada por más de 30 años para agrupar y caracterizar a diversos taxa del género (Klappenbach 1968, Braun \& Braun 1979, Prigioni \& Langone 1986, Caramaschi \& Cruz 2002).

El objetivo del presente trabajo es establecer las diferencias histológicas del tegumento de la región cefálica entre especies del género Melanophryniscus pertenecientes al grupo stelzneri (sin tumefacción frontal) y especies pertenecientes al grupo tumifrons (con tumefacción frontal). De esta forma se pretende aclarar la estructura de la tumefacción frontal y discutir su posible función.

\section{MATERIALES Y MÉTODOS}

El estudio histológico se realizó usando ejemplares fijados y preservados en colecciones zoológicas. Si bien esto pudo haber determinado una disminución en la calidad del material analizado fue un hecho inevitable dada la dificultad de colectar ejemplares vivos de varias de las especies analizadas. Se examinaron ejemplares adultos de las siguientes especies del grupo stelzneri: Melanophryniscus atroluteus (ZVCB 2315), M. aff. cupreuscapularis,

\footnotetext{
${ }^{1}$ ZVCB: Colección Zoología Vertebrados, Facultad de Ciencias, Universidad de la República, Uruguay; MNHN: Museo Nacional de Historia Natural, Uruguay; MCP: Museu de Ciencias da Pontificia Universidade Católica de Porto Alegre.
}

(ZVCB 10250), M. klappenbachi, (MNHN 677) y M. montevidensis (ZVCB 10251); del grupo tumifrons se analizaron: $M$. orejasmirandai (ZVCB 10252), y M. cambaraensis $(\mathrm{MCP} 2783)^{1}$.

Se removió la piel y las muestras obtenidas fueron deshidratadas e incluidas en paraplast según técnicas convencionales para microscopia óptica (Bancroft \& Stevens 1996). Se realizaron cortes transversales seriados, de 10 $\mu \mathrm{m}$, desde el extremo anterior del hocico hasta la línea media entre los ojos, los cuales se colorearon con Hematoxilina-Eosina y Tricromo de Milligan (Pressnell \& Schreibman 1997). Las observaciones fueron realizadas usando un microscopio Olympus BX40 con ocular 10X y objetivos 4X, 10X, 20x, 40x y 100x. Los registros fotográficos se obtuvieron con una cámara digital Sony CCD-IRIS. Para cada especie se analizó en primera instancia la histología de la región cefálica lateral, la cual se comparó posteriormente con la histología de la región frontal. Cabe mencionar que la tumefacción frontal no es un rasgo que presente dimorfismo sexual (J.A. Langone observación personal).

\section{RESULTADOS}

\section{Características generales del tegumento}

La epidermis consta de tres estratos (Fig.1). El estrato córneo consiste de una capa delgada de queratina que cubre de forma irregular las células del estrato intermedio. El estrato intermedio consta de dos a cinco capas de células; en algunos casos se observaron gránulos de melanina en el citoplasma de estas células. El estrato germinativo es un epitelio monoestratificado de células cilíndricas. La región cefálica exhibe engrosamientos epidérmicos, determinados por un aumento en número y volumen de las células del estrato intermedio y también por un engrosamiento del estrato córneo. Entre la epidermis y la dermis existe una delgada lámina basal compuesta por fibras colágenas.

La dermis consta de dos estratos: el estrato laxo o esponjoso y el estrato compacto. El primero consiste de una matriz irregular de fibras colágenas en la cual se sitúan glándulas, melanóforos, células conectivas, fibroblastos, miocitos, y vasos sanguíneos. Los melanóforos se disponen principalmente debajo de la lámina 
basal y alrededor de las glándulas presentes en la dermis. Se observan dos tipos de glándulas alveolares simples: glándulas mucosas y glándulas granulares. Las primeras son de forma redondeada y poseen paredes celulares definidas formadas por células cilíndricas. Las glándulas granulares tienden a ser ovaladas, gene- ralmente de mayor tamaño que las anteriores y poseen paredes sincitiales. Cabe notar que en la dermis de las especies del grupo stelzneri las glándulas mucosas ocupan la región más apical del estrato laxo, mientras que en la dermis de los especimenes del grupo tumifrons ambos tipos de glándulas se encuentran a la
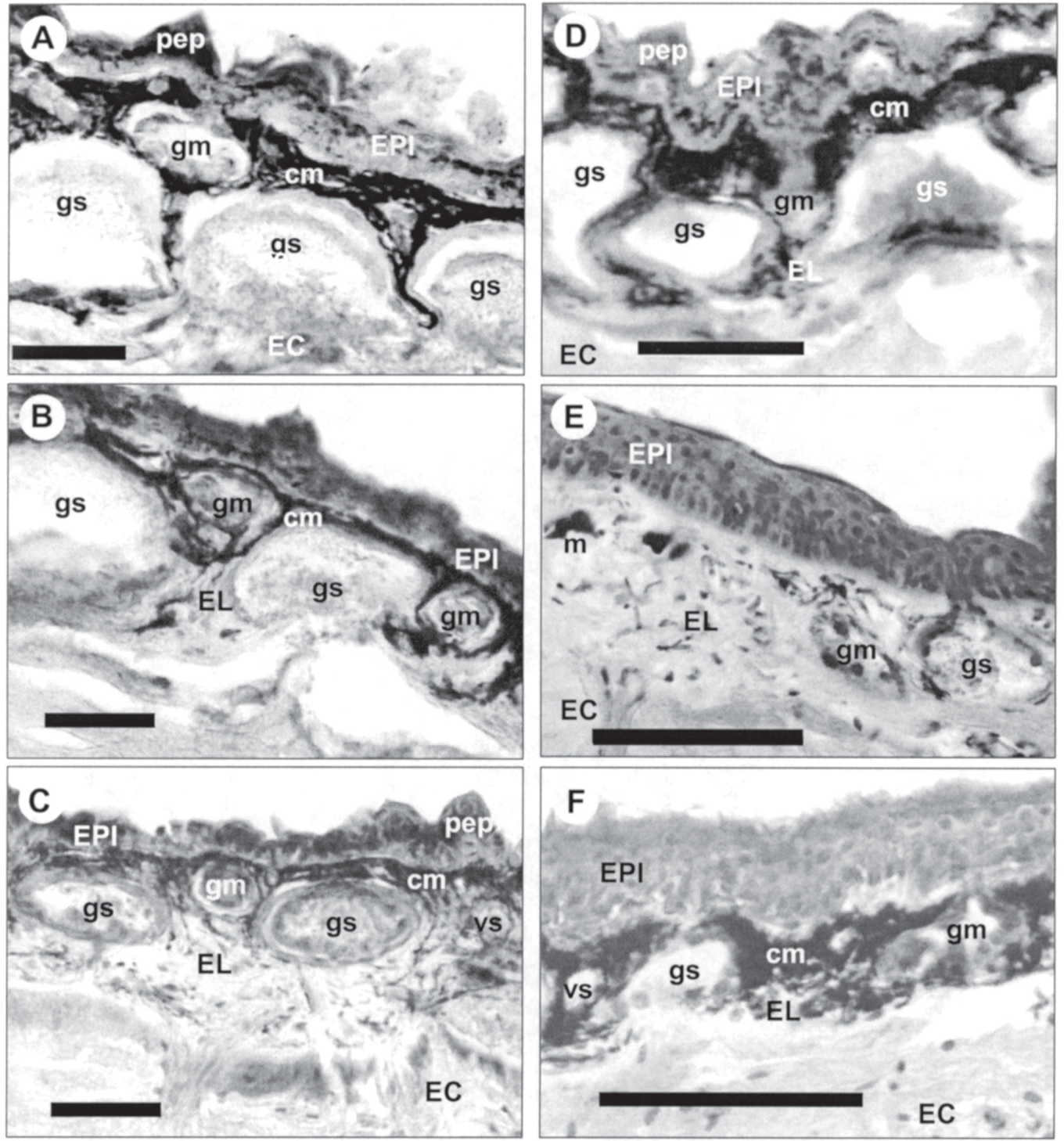

Fig. 1: Cortes transversales del tegumento de la región cefálica lateral de: (A) M. atroluteus, (B) M. cupreuscapularis, (C) M. klappenbachi, (D) M. montevidensis, (E) M. orejasmirandai, y (F) $M$. cambaraensis: $\mathrm{EPI}=$ epidermis, pep $=$ engrosamiento epidérmico, $\mathrm{cm}=$ capa de melanóforos, $\mathrm{m}=$ melanóforo, vs = vasos sanguíneos, $\mathrm{EL}=$ estrato laxo, $\mathrm{EC}=$ estrato compacto, gs = glándula serosa, $\mathrm{gm}=$ glándula mucosa, $\mathrm{HIPO}=$ hipodermis. Barra $=100 \mu \mathrm{m}$.

Skin transversal sections of lateral head region of : (A) M. atroluteus, (B) M. cupreuscapularis, (C) M. klappenbachi, (D) M. montevidensis, (E) M. orejasmirandai, and (F) $M$. cambaraensis: $\mathrm{EPI}=$ epidermis, pep $=$ epidermis peak, $\mathrm{cm}=$ melanophore layer, $\mathrm{m}=$ melanophores, $\mathrm{vs}=$ blood vessels, $\mathrm{EL}=$ stratum spongiosum, $\mathrm{EC}=$ stratum compactum, $\mathrm{gs}=$ serous glands, $\mathrm{gm}=$ mucous glands, $\mathrm{HIPO}=$ hypodermis. $\mathrm{Bar}=100 \mu \mathrm{m}$. 
misma profundidad. El estrato compacto está constituido por fibras colágenas ordenadas en bloques ondulados, de compactación variable y orientación paralela a la epidermis; entre estos bloques es común observar fibras musculares y capilares sanguíneos.

\section{Modificaciones en la región frontal}

Las especies del grupo stelzneri no mostraron cambios entre la histología de las regiones de piel cefálica lateral y frontal. La relación de espesores entre la dermis y epidermis (D/E) permaneció constante entre ambas regiones, abarcando valores que oscilaron entre seis y nueve según la especie analizada. Por el contrario, las especies pertenecientes al grupo tumifrons (Fig. 2), exhiben una epidermis de espesor homogéneo, con menor densidad de engrosamientos epidérmicos, y un grueso estrato laxo de la dermis (valores de D/E de aproximadamente 20). El aumento en el grosor del estrato laxo se correlaciona con el aumento del tamaño de las glándulas granulares (de 50-100 $\mu \mathrm{m}$ a más de $500 \mu \mathrm{m}$ ), las cuales adquieren una forma alargada, con su eje mayor perpendicular a la superficie. El incremento de las glándulas granulares determina la acumulación de glándulas mucosas en el sector apical del estrato laxo (como se observa en las especies del grupo stelzneri), y un mayor ordenamiento y compac- tación de las fibras colágenas en el estrato compacto de la dermis. Además se observa un aumento en la cantidad de melanóforos rodeando a las glándulas granulares.

\section{DISCUSIÓN}

El cambio filogenético de glándulas unicelulares epidérmicas en peces óseos a glándulas multicelulares dérmicas en anfibios, determinó un notorio incremento de la flexibilidad secretora en estos últimos (Delfino et al. 1998). Al respecto, la piel de los anuros sintetiza más aminas biogénicas, péptidos y esteroides que los demás grupos de vertebrados (Daly et al. 1987, Barthalmus 1994). No obstante, existe una composición tegumentaria básica que permanece relativamente constante en todos los anfibios (Elias \& Shapiro 1957, Duellman \& Trueb 1994, Toledo \& Jared 1995). En este sentido, la histología del tegumento de la región cefálica lateral de las seis especies consideradas, se corresponde con descripciones existentes para el género Melanophryniscus (e.g., Santos 1979).

La histología del tegumento de la región frontal indica claramente que la tumefacción que caracteriza al grupo tumifrons es una macroglándula (según Toledo \& Jared 1995). Es decir, esta estructura consiste en una agrupación de glándulas granulares de gran tamaño y
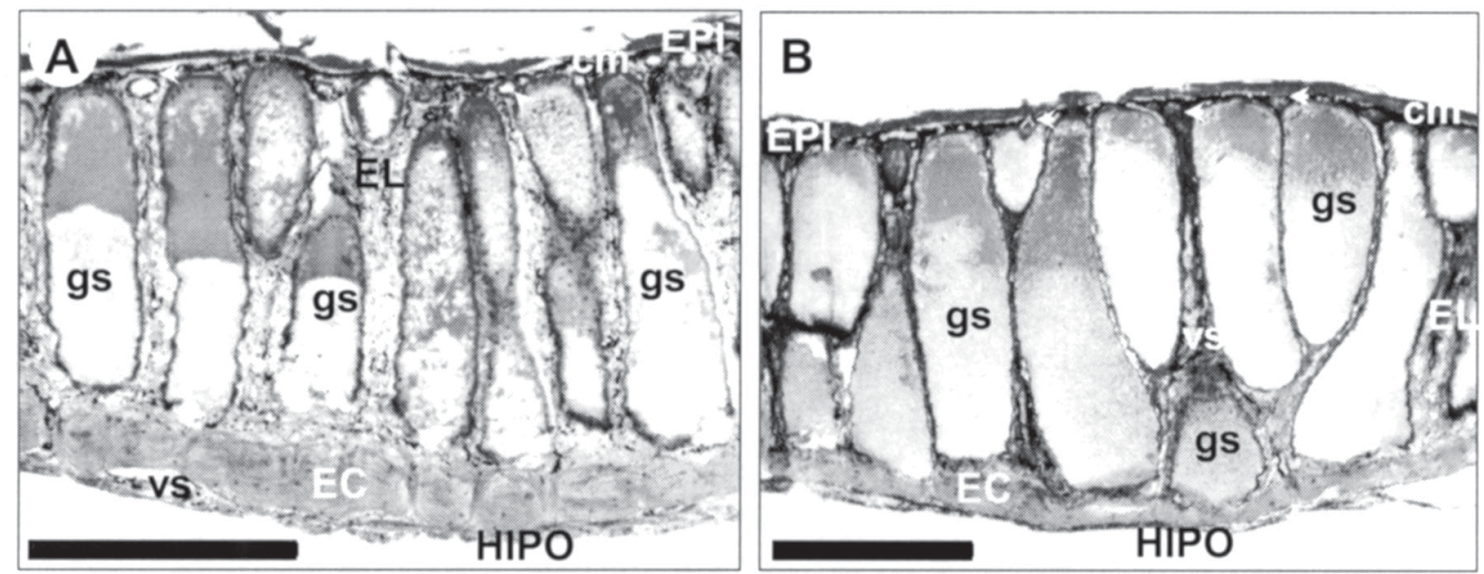

Fig. 2: cortes transversales de tegumento de la región cefálica frontal de (A) M. orejasmirandai, y (B) M. cambaraensis; flechas = glándulas mucosas; otras abreviaturas como en Fig. 1. Barra $=500 \mu \mathrm{m}$.

Skin transversal sections of frontal head region of (A) M. orejasmirandai, y (B) M. cambaraensis; arrows = mucous glands; others abbreviation as in Fig. 1. Bar $=500 \mu \mathrm{m}$. 
disposición contigua, similar a otras macroglándulas presentes en la dermis de anuros (e.g., Blaylock et al. 1976, Crook \& Tyler 1981, Jacob et al. 1985, Lacombe et al. 2000). Para el caso particular de los bufónidos, se han descrito macroglándulas de estructura histológica similar a la tumefacción frontal, como ser las glándulas parotoides y glándulas tibiales (Hostetler \& Cannon 1974, Cannon \& Hostetler 1976). Dado que hasta el momento no se había conocido la estructura glandular de la tumefacción frontal, proponemos que dicho rasgo anatómico sea designado como macroglándula frontal. Esta distinción es importante ya que el género Melanophryniscus, a pesar de tener toxinas en la piel como otros bufonidos (Daly et al. 1984) no posee otras macroglándulas.

La secreción de las glándulas granulares de los anfibios consiste comúnmente en sustancias tóxicas o repelentes (Stebbins \& Cohen 1995) de origen diverso, como ser péptidos bioactivos, esteroides, alcaloides y sus derivados (Houck \& Sever 1994). Para el caso particular del género Melanophryniscus se han descrito diversos alcaloides lipofílicos tóxicos (Daly et al. 1984, 1987, Garrafo et al. 1993). Considerando la posición que la macroglándula frontal ocupa en el cuerpo y el elevado número de glándulas granulares que la constituyen es posible que dicha estructura tenga una función defensiva. Observaciones de laboratorio realizadas en ejemplares de $M$. orejasmirandai indican la producción de una secreción lechosa y viscosa por parte de la macroglándula frontal, cuando los individuos se encontraban en estado de estrés por presencia de un depredador ofidio (D. Arrieta comunicación personal). Estudios etológicos y bioquímicos adicionales son necesarios para corroborar dicha función.

\section{AGRADECIMIENTOS}

A Marco Di Bernardo por suministrar el ejemplar de M. cambaraensis y permitir su disección; a Gary Radice y Nibia Berois por la asistencia técnica, comentarios y sugerencias. D.E.N. agradece a Arley Camargo por el tiempo compartido en Richmond y financiamientos otorgados por el Programa de Desarrollo de las Ciencias Básicas (PEDECIBA, Uruguay), la Universidad de Richmond (USA), la Dirección General de Postgrado de la Pontificia Universi- dad Católica de Chile (Chile), CASEB (Programa 1), y la Comisión Nacional de Investigación Científica y Tecnológica (CONICYT, Chile).

\section{LITERATURA CITADA}

BANCROFT JD \& A STEVENS (1996) Theory and practice of histological techniques. Fourth Edition. Churchill Livingston, New York, New York, USA. $766 \mathrm{pp}$.

BARTHALMUS GT (1994) Biological roles of amphibian skin secretions. En: Heatwole H \& GT Barthalmus (eds) Amphibian biology (volume 1): the integument: 382-410. Surrey Beatty \& Sons, Chipping Norton, Australia.

BIRABEN M (1929) Sobre la estructura histológica de las glándulas venenosas de la piel de anfibios de la República Argentina. Physis (Argentina) 9: 416-420.

BLAYLOCK LA, R RUIBAL \& K PLATT-ALOIA (1976) Skin structure and wiping behavior of Phyllomedusine frogs. Copeia 1976: 283-295.

BRAUN PC \& CAS BRAUN (1979) Nova espécie de Melanophryniscus Gallardo, 1961 do Estado do Rio Grande do Sul, Brasil. Iheringia Zoología (Brasil) 54: 16-20.

CANNON MS \& JR HOSTETLER (1976) The anatomy of the parotoid gland in Bufonidae with some histochemical findings. II Bufo alvarius. Journal of Morphology 148: 137-160.

CARAMASCHI U \& CAG CRUZ (2002) Taxonomic status of Atelopus pachyrhynus Miranda-Ribeiro, 1920. Redescription of Melanophryniscus tumifrons (Boulenger, 1905), and descriptions of two new species of Melanophryniscus from the State of Santa Catarina, Brazil (Amphibia, Anura, Bufonidae). Arquivos do Museu Nacional (Brasil) 60: 303-314.

CROOK GA \& MJ TYLER (1981) Structure and function of the tibial gland of the australian frog Limnodynastes dumerili Peters. Transactions of the Royal Society of South Australia 105: 49-52.

DALY JW, RJ HIGHET \& CW MYERS (1984) Occurrence of skin alkaloids in non-dendrobatid frogs from Brazil (Bufonidae), Australia (Myobatrachidae) and Madagascar (Mantellinae). Toxicon 22: 905-919.

DALY JW, CW MYERS \& N WHITTAKER (1987) Further classification of skin alkaloids from neotropical poison frogs (Dendrobatidae) with a general survey of toxic / noxious substances in the amphibia. Toxicon 25: 1023-1095.

DELFINO G, R BRIZZI, R KRACKE-BERNDORFF \& B ÁLVAREZ (1998) Serous gland dimorphism in the skin of Melanophryniscus stelzneri (Anura: Bufonidae). Journal of Morphology 237: 19-32.

DUELLMAN WE \& L TRUEB (1994) Biology of Amphibians. The Johns Hopkins University Press, London, United Kingdom. 670 pp.

ELIAS H \& J SHAPIRO (1957) Histology of the skin of some toads and frogs. American Museum Novitates 1819: 1-27.

GARRAFO HM, TF SPANDE, JW DALY, A BALDESSARI \& EG GROSS (1993) Alkaloids from bufonid toads (Melanophryniscus): decahydroquinolines, pumiliotoxins and homopumiliotoxins, indolizidines, pyrrolizidines, and quinolizidines. Journal of Natural Products 56: 357-373.

HEATWOLE H \& GT BARTHALAMUS (1994) 
Amphibian biology (volume 1): the integument. Surrey Beatty \& Sons, Chipping Norton, Australia. $418 \mathrm{pp}$.

HOSTETLER JR \& MS CANNON (1974) The anatomy of the parotoid gland in Bufonidae with some histochemical findings. I Bufo marinus. Journal of Morphology 142: 225-240.

HOUCK LD \& DM SEVER (1994) Role of the skin in reproduction and behaviour. En: Heatwole $\mathrm{H} \& \mathrm{GT}$ Barthalmus (eds) Amphibian biology (volume 1): the integument: 351-381. Surrey Beatty \& Sons, Chipping Norton, Australia.

JACOB JS, JJ GREENHAW, JV PLUMMER \& JM GAY (1985) Pectoral glands of Scaphiopus and Megophrys. Journal of Herpetology 19: 419-420.

KLAPPENBACH, MA (1968) Notas herpetológicas IV. El género Melanophryniscus (Amphibia, Salientia) en el Uruguay, con la descripción de dos nuevas especies. Comunicaciones Zoológicas del Museo de Historia Natural de Montevideo (Uruguay) 9: 1-12.

LACOMBE C, C CIFUENTES-DÍAZ, I DUNIA, M AUBER-THOMAY, P NICOLAS \& M AMICHE (2000) Peptide secretion in the cutaneous glands of South American tree grog Phyllomedusa bicolor. An ultraestructural study. European Journal of Cell Biology 79: 631-641.

MANGIONE S \& MF ALCAIDE (1994) Histología de la piel del parche pélvico, en referencia a piel dorsal y venral en Melanophyrniscus rubriventris subconcolor (Anura, Bufonidae). Boletín de la Aso- ciación Herpetológica Argentina 10: 28-30.

PRESSNELL JK \& MP SCHREIBMAN (1997) Humason's animal tissue techniques. Fifth edition. The Johns Hopkins University Press, Baltimore, Maryland, USA. 572 pp.

PRICE TD, A QVARNSTROM \& DE IRWIN (2003) The role of phenotypic plasticity in driving genetic evolution. Proceedings of the Royal Society of London B 270: 1433-1440.

PRIGIONI CM \& JA LANGONE (1986) Melanophryniscus orejasmirandai n. sp., un nuevo Bufonidae (Amphibia, Anura) de Uruguay con una clave para las especies del grupo tumifrons. Comunicaciones Zoológicas del Museo de Historia Natural de Montevideo (Uruguay) 11: 1-11.

SANTOS AB (1979) Estudo Histologico do integumento dorsal de Melanophryniscus stelzneri atroluteus (Anura-Amphibia). Acta Biologica Leopoldensia 1: 289-312.

STEBBINS RC \& NW COHEN (1995) A natural history of amphibians. Princeton University Press, Princeton, New Jersey, USA. 316 pp.

TOLEDO RC \& C JARED (1993) Cutaneous adaptations to water balance in amphibians. Comparative Biochemistry and Physiology 105A: 593-608.

TOLEDO RC \& C JARED (1995) Cutaneous granular glands and amphibian venoms. Comparative Biochemistry and Physiology 111A: 1-29. 